\title{
RECRYSTALLIZATION BEHAVIORS IN THE PRODUCTION OF STRUCTURAL STEELS*
}

Douglas Glenn Stalheim ${ }^{1}$

\begin{abstract}
The uniformity of the final cross sectional microstructure and subsequent final mechanical properties and shape of structural steels is dependent on the type or types of recrystallization behaviors that are created. There are three main recrystallization behaviors that are the foundation of the metallurgy created during production. Alloy design and subsequent processing design strategy drive how much of each recrystallization behavior is created through the cross section of the product. Carbon manganese steels create only one basic behavior while the addition of microalloys, in particular niobium, can add two additional behaviors. The behaviors are intertwined with each other and build upon each other. The three behaviors are Type I, static recrystallization, Type II, no-recrystallization, and Type III, metadynamic/dynamic recrystallization. How much of each behavior is generated through the cross section through the production influences the final transformed microstructure and corresponding mechanical properties and shape performance. This paper will discuss the three behaviors, how they are generated, how to identify which behavior is occurring through modeling or actual mill data. Actual examples from production will be discussed.
\end{abstract}

Keywords: Recrystallization; Behaviors; Niobium; Microstructure. 


\section{INTRODUCTION}

The fundamental metallurgy that sets the stage for the final cross sectional microstructure and hence the mechanical properties and shape performance of structural steels is related to the final transformed cross sectional ferrite grain size and distribution. $[1,2]$ The final transformed ferrite grain size and distribution is driven by the recrystallization behaviors that are generated by the alloy and process design. There are three recrystallization behaviors, sometimes referred to as "softening mechanisms", which can be generated, depending on alloy design during steel processing. One, two or three of the recrystallization behaviors can be developed or even some combination of the three behaviors. The three recrystallization behaviors/"softening mechanisms" are Type I, static recrystallization, Type II, no recrystallization and Type III, metadynamic/dynamic recrystallization (simply Type III can be a mixture of Type I and Type II occurring at the same time), Figure 1. [3]

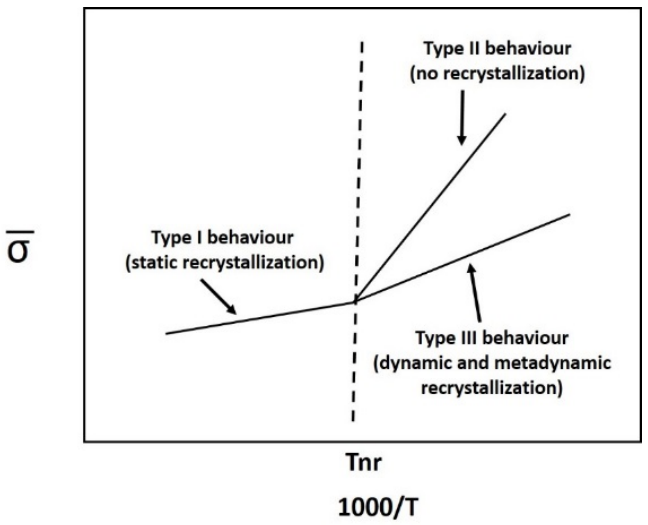

Figure 1. Illustration of Three Recrystallization Behaviors Types vs. Flow Stress

The graph in Figure 1 represents the fundamental recrystallization behaviors above and below the no-recrystallization temperature (Tnr) seen in structural steels that needs to be understood to properly design the alloy and processing to achieve the desired end microstructure, mechanical properties and final shape. However, Tnr does not typically represent one single temperature as seen in Figure 1, but covers a range of temperatures where one has 95\% recrystallization occurring (RLT, recrystallization lower temperature) and $5 \%$ recrystallization occurring (RST, recrystallization stop temperature), Figure 2 .

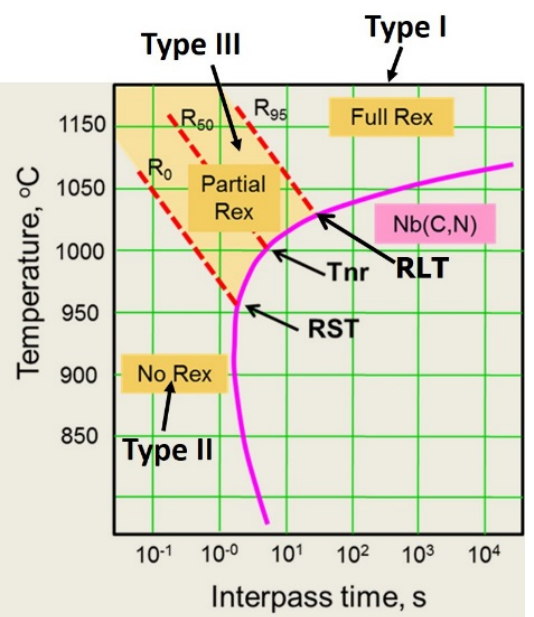

Figure 2. Illustration of recrystallization behavior Types vs. Tnr for $0.030 \% \mathrm{Nb}$ [4] - DGS modified. 
For a Tnr temperature to occur and Type II or Type III behavior to develop microalloys must be part of the alloy design along with proper processing design. In particular, niobium has a large influence on Type II and Type III behavior as it affects the recrystallization behavior during rolling deformation. The other microalloys of vanadium and titanium have a much lesser effect on the recrystallization behaviors due to the low processing temperatures where they are effective, Figure 3. [5] Most structural steel rolling is conducted at temperatures $>850^{\circ} \mathrm{C}$, as illustrated by the red line in Figure 3, which explains the significant influence of niobium on the formation of Type II and Type III recrystallization behaviors.

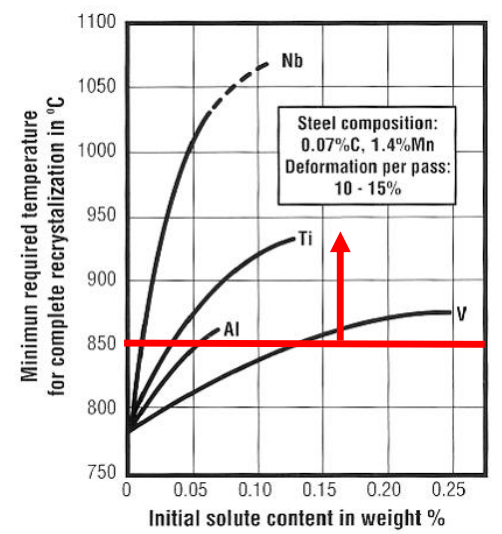

Figure 3. Illustration of microalloy effect on recrystallization stop temperature. Most structural steel rolling is conducted at temperatures $>850^{\circ} \mathrm{C}$ as highlighted by the red line/arrow.

\section{RECRYSTALLIZATION BEHAVIOR DEVELOPMENT}

The proper development of the cross sectional recrystallization behavior drives the final cross sectional transformed grain size which is the foundation for all of the metallurgy, microstructure, mechanical properties and shape.[6] Alloy and process design drive which or combination of which recrystallization behaviors occur through the cross section. Final transformed grain size for most structural steels can range from ASTM $5(65 \mu \mathrm{m})$ to ASTM $12(5.6 \mu \mathrm{m})$ or beyond during processing. Type I static recrystallization behavior typically results in final transformed ferrite grain size in the ASTM 5-9 range depending on the level of optimization of the rolling process. Type III metadynamic/dynamic recrystallization behavior typically results in a mixture of final cross sectional transformed ferrite grain size in the ASTM 8-10 or 11 range again depending on the level of the optimization of the rolling process for the alloy design. Type II no-recrystallization can result in the absolute finest transformed ferrite grain size in the ASTM 10-12 or beyond range depending on the level of optimization of the rolling process for the alloy design. Figure 4 illustrates how the three recrystallization behaviors can affect the final transformed ferrite ASTM grain size. 
ASTM E112 Grain Size Number



Figure 4. Illustration of the three recrystallization behavior effect on the final transformed ferrite ASTM grain size. Yellow bracket illustrates Type I static recrystallization behavior. Red bracket illustrates Type III metadynamic/dynamic behavior. Brown bracket illustrates Type II no-recrystallization behavior.

\subsection{Type I - Static Recrystallization}

Type I static recrystallization is the starting point and can be the ending point of the recrystallization behavior developed in the cross section of structural steel depending on alloy design and process design. If no microalloy, particularly niobium, is not in the alloy design then Type I static recrystallization will drive the final transformed ferrite grain size. If niobium is added to the alloy design then how well the Type I static recrystallization behavior is processed will be the beginning point for the development of either Type II no-recrystallization or Type III metadynamic/dynamic recrystallization behaviors. How much of either Type II or Type III that is developed at the end of the rolling will be driven by the niobium addition and process design (deformations, interpass times, temperatures, etc.).

Type I static recrystallization consists of a deformation of reheated equiaxed austenite grains, for non-CSP type operations, followed by a recovery/recrystallization of the deformed austenite grains into slightly smaller equiaxed austenite grains as illustrated in Figure 5.

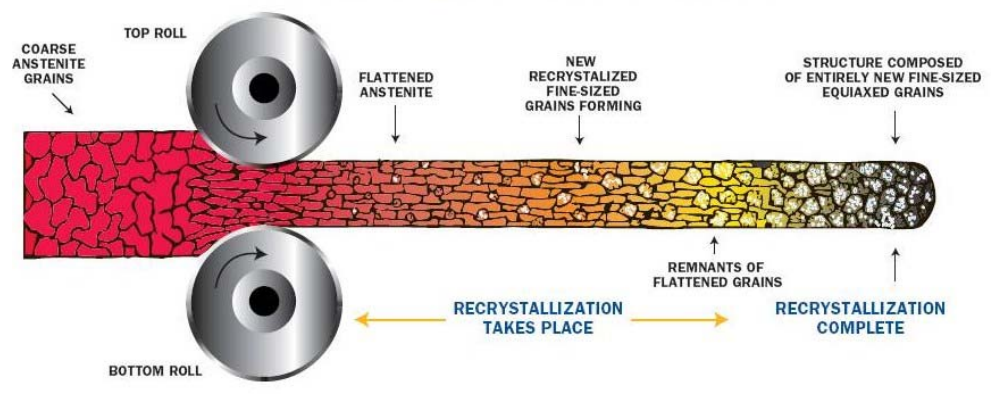

Figure 5. Illustration of Type I static recrystallization process

Regardless if the Type I static recrystallization behavior is being used to produce the final transformed ferrite grain size singly or as the starting base in combination with Type III or Type II behavior it should be the goal to attempt to achieve a final Type I recrystallized austenite grain size in the $30-50 \mu \mathrm{m}$ range which is about as small as 
can be practically accomplished in a production environment. To achieve this austenite grain size the process has to be optimized to take a single significant deformation, preferably in the $15 \%$ plus range, in the $50-60 \%$ total deformed cross section, Figure 6. [7]

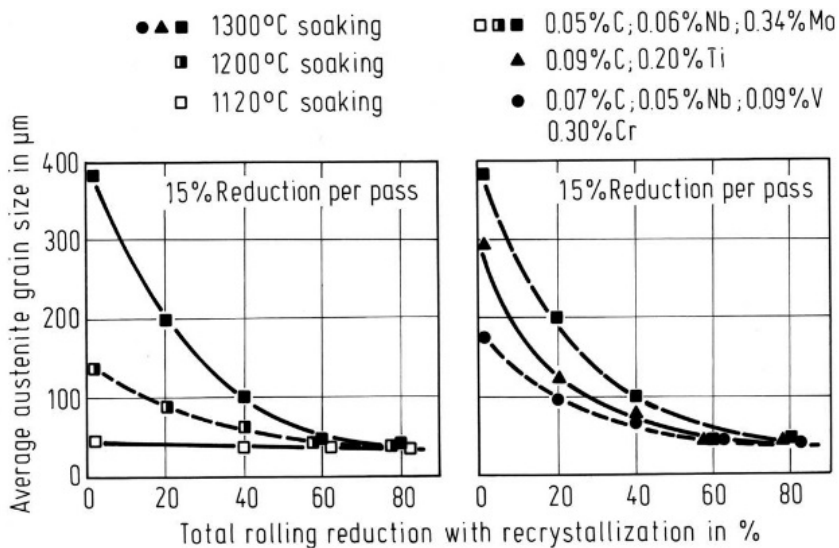

Figure 6. Example of influence of total Type I static recrystallization deformation on optimized final austenite grain size with $15 \%$ deformation.

\subsection{Type II - No-Recrystallization}

Type II no-recrystallization behavior will result in the absolute finest transformed ferrite grain size which will positively affect strength, elongation and toughness. This is accomplished by imparting deformation below the RST temperature, norecrystallization temperature as seen in previous Figure 2.

When deformation of the austenite grain is done below the RST temperature there is no recovery/recrystallization of the austenite grain, typically referred to as flattening or "pancaking", Figure 7, due to grain pining by $\mathrm{Nb}$ solute drag and/or fine $\mathrm{Nb}$ precipitates that come out of solution with the per pass deformation.

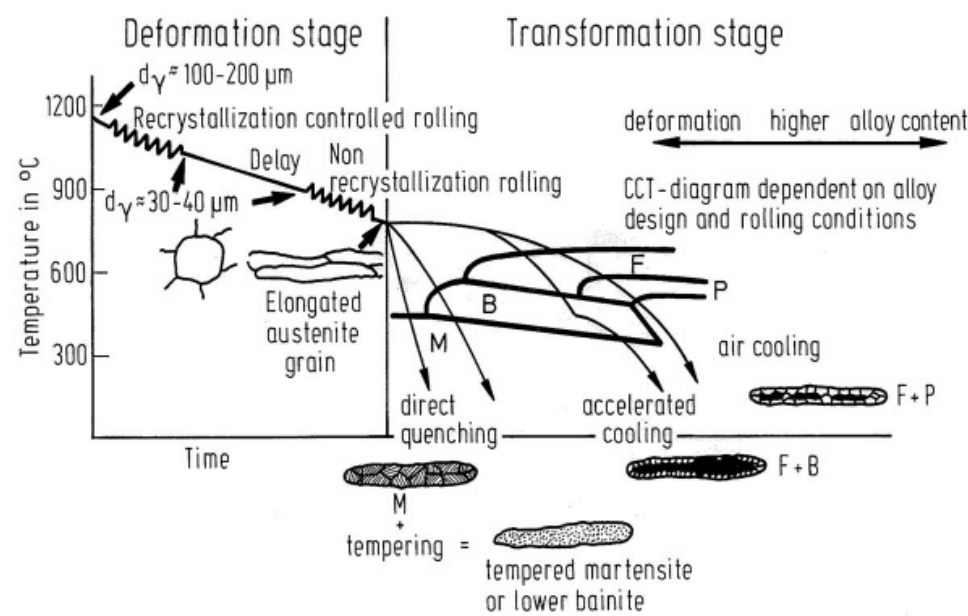

Figure 7. Illustration of Type II no-recrystallization behavior deformation, elongation/pancaking of the austenite grain.

Figure 8 shows the comparison of Type I static recrystallization austenite grain size formation vs. Type II no-recrystallization austenite grain size formation. 


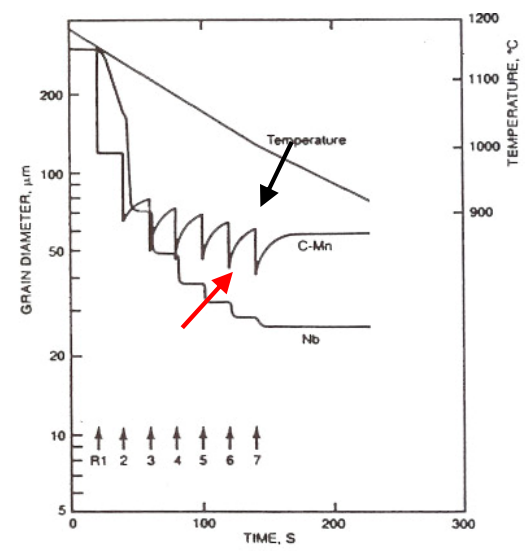

Figure 8. Comparison of austenite grain size vs. Type I, black arrow, or Type II, red arrow, behavior [8]

How fine the final transformed ferrite grain will become will be driven by the starting Type I statically recrystallized formed austenite grain, the total amount of Type II deformation, Figure 9, and the per pass deformation percentage/strain accumulation achieved.

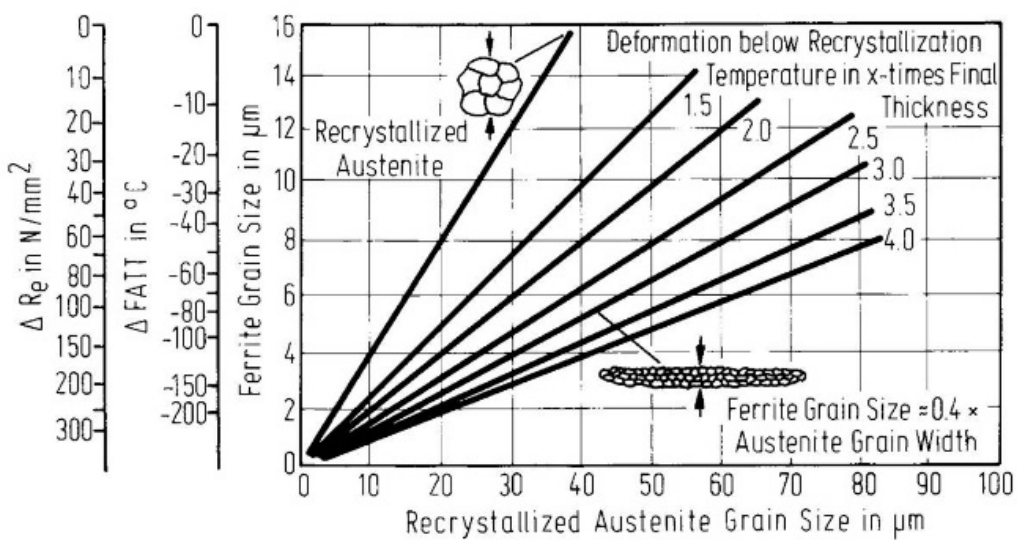

Figure 9. Starting Type I recrystallized austenite grain size, x-axis, vs. final transformed ferrite grain size/strength/fracture toughness transition temperature for various volume fractions of Type II norecrystallization behavior labeled "Deformation below Recrystallization Temperature in x-times Final Thickness".

Figure 10 shows the effect of various percent total pure Type II no-recrystallization behavior on the charpy fracture toughness transition temperature.

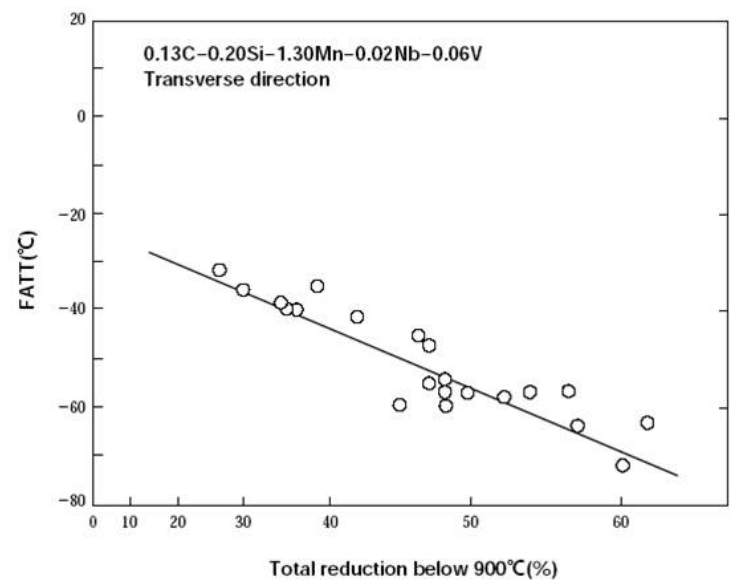

Figure 10. Effect of pure Type II no-recrystallization behavior percent vs. charpy toughness transition temperature 


\subsection{Type III - Metadynamic/Dynamic Recrystallization}

When the steel is deformed between the RLT and the RST temperature, after first being deformed in the Type I static recrystallization temperature range, a mixture of Type I static recrystallization behavior and Type II no-recrystallization behavior can occur. In addition, if the niobium content is insufficient and/or the speed of deformation very high such as in very light gauge hot strip rolling, dynamic recrystallization can occur. Dynamic recrystallization results in recovery/recrystallization of the austenite grain rather than the expected "pancaking" of the austenite grain in Type II no-recrystallization behavior, resulting in a larger grain vs. a finer grain. Regardless if a mixture of Type I/Type II recrystallization behavior and/or dynamic recrystallization occurs, the end result is a mixed size distribution of transformed ferrite grains through the cross section. The mixed distribution will be overall finer than that of pure Type I static recrystallization generally resulting in improved mechanical properties. However, pure Type II norecrystallization behavior after proper Type I static recrystallization behavior results in the optimum mechanical property performance.

\section{UTILIZATION OF RECYRSTALLIZATION BEHAVIORS}

It is well documented that improved strength and ductility (elongation/toughness) comes from a uniformly fine cross sectional grain size, Figure 11. [9,6]

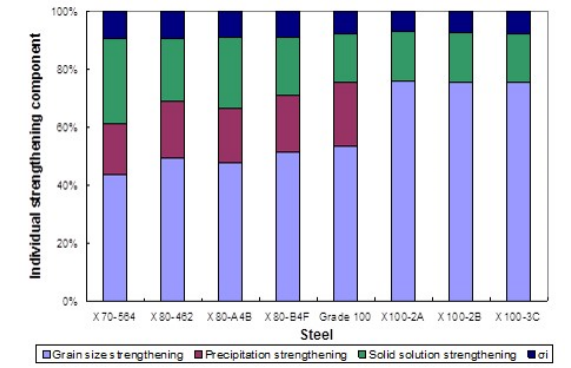

Grain size component of overall strength

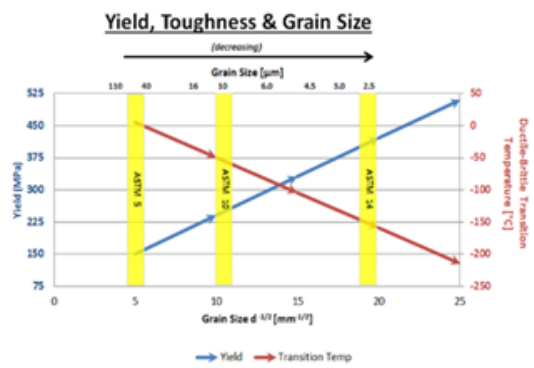

Grain size vs. strength and toughness



ASTM Grain Size vs. Strength and Elongation vs. Recrystallization Behavior Type, Courtesy of Michael Wright, Modern Metal Solutions Pte, Ltd.

Figure 11. Examples of effect of grain size on strength and ductility

Therefore if optimum strength and ductility are to be realized, proper development of the three recrystallization behaviors is required. For optimum cross sectional grain size a combination of Type I behavior and/or some combination of Types II and III behavior are required. Type I plus Type II will give the finest cross sectional grain size. As the volume fraction of Type II behavior is reduced to Type III behavior the grain size will start to become non-uniform through the cross section.

For optimum cross sectional grain size uniformity Type I static recrystallization behavior total volume fraction should be between $50-60 \%$. This will result in an 
austenite grain size in the 30-50 $\mu \mathrm{m}$ range (assuming reasonable per pass reductions). Increasing the volume fraction of Type I static recrystallization behavior does not further refine the austenite grain size significantly as seen in previous Figure 6. An austenite grain size in the 30-50 $\mu \mathrm{m}$ range will result in an ASTM transformed ferrite grain size of approximately ASTM 8-9.

This sets the "stage" for how fine the final austenite grain size will become with either Type II no-recrystallization or Type III metadynamic/dynamic recrystallization behavior deformations. Utilizing niobium's ability to retard/stop recrystallization from occurring will then start the promotion of Type II and Type III behavior. This is dependent on how much niobium is in the solution during deformation and the overall total and per pass deformations that are taken.

There are several tools that can be used to help in setting up the rolling process and then evaluating what type of recrystallization behavior(s) that actually occurred. Equations that have been developed to calculate the non-recrystallization temperature, Tnr, based on alloy designs can help in determining the starting temperature for either Type II or Type III behavior. Equations by Boratto/Barbosa (Equation 1), Boratto/Barbosa DGS Modified (Equation 2) or Bai (Equation 3) can be used: $[10,11]$

$$
\begin{array}{r}
\mathrm{Tnr}=887+464 \mathrm{C}+(6445 \mathrm{Nb}-644 \sqrt{\mathrm{Nb}})+(732 \mathrm{~V}-230 \sqrt{\mathrm{V}})+890 \mathrm{Ti}+363 \mathrm{Al}-357 \mathrm{Si} \\
\mathrm{Tnr}=887+464 \mathrm{C}+\left(6445\left(0.80^{*} \mathrm{Nb}\right)-644 \sqrt{ }\left(0.80^{*} \mathrm{Nb}\right)\right)+\left(732\left(0.80^{*} \mathrm{~V}\right)-\right. \\
\left.230 \sqrt{ }\left(0.80^{*} \mathrm{~V}\right)\right)+890\left(0.80^{*} \mathrm{Ti}\right)+363\left(0.80^{*} \mathrm{Al}\right)-357\left(0.80^{*} \mathrm{Si}\right) \\
\operatorname{Tnr}=\left(174 \log \left(\mathrm{Nb}^{*}\left(\mathrm{C}+12 / 14^{*} \mathrm{~N}\right)\right)+1444\left({ }^{\circ} \mathrm{C}\right)\right)-75\left({ }^{\circ} \mathrm{C}\right)
\end{array}
$$

In addition, recently developed metallurgical models can also be used to demonstrate the type of recrystallization behaviors generated during rolling, Figure 12.

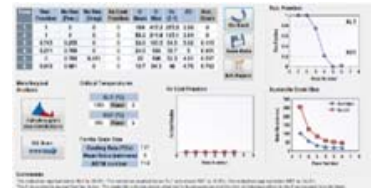

CSP metallurgical model showing recrystallization behavior of the austenite grain for each rolling pass. Developed by CEIT/CBMM.

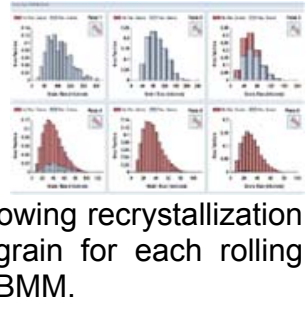

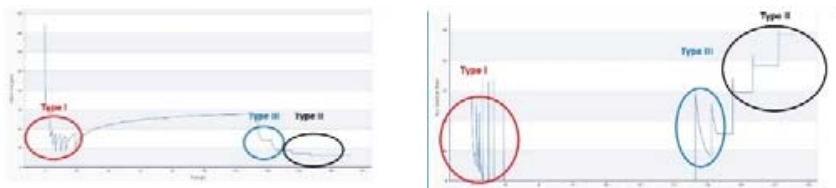

Plate metallurgical model showing austenite grain size evolution, strain accumulation and recrystallization behavior by pass. Developed by CBMM

Figure 12. Examples of metallurgical models used to show grain size/recrystallization behavior types

Using these models, the effect of niobium on austenite grain size distribution per pass along with the recrystallization behaviors developed can be easily seen, Figure 13.

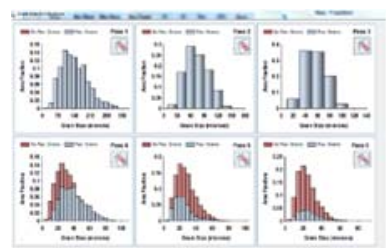

$0.020 \%$ Nb, Type I - Pass 1-3, Type III - Pass 3-6

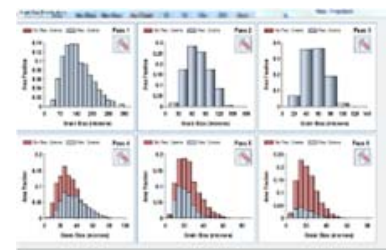

$0.030 \% \mathrm{Nb}$, Type I - Pass 1-3, Type III - Pass 3-6



$0.050 \% \mathrm{Nb}$, Type I - Pass 1-2, Type III - Pass 3-4, Type II - Pass 5-6

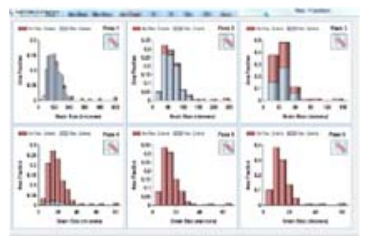

$0.070 \% \mathrm{Nb}$, Type I Pass 1, Type III - Pass 2-3, Type II - Pass 4-6

Figure 13. Metallurgical modeling of various niobium levels and resultant austenite recrystallization behaviors per pass

Along with these metallurgical models which can be used with theoretical rolling data or actual mill data, mean flow stress can also be used from the actual production data from the mill to show where the Tnr occurred and what type of recrystallization 
behavior is occurring with each pass. Each recrystallization behavior as a general slope as seen in Figure 1 and generates a range of mean flow stress values per pass. Type I static recrystallization behavior generates mean flow stress in the 70$200 \mathrm{MPa}$ range depending on alloy, temperature, dimension, etc. Type II norecrystallization behavior generates mean flow stresses in the 200-400 MPa range depending on alloy, temperature, dimension, etc. and Type III metadynamic/dynamic recrystallization behavior generates mean flow stress in the 150-250 MPa range, Figure 14.

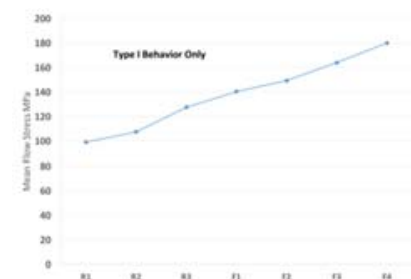

Type I Static Recrystallization Only, C/Mn Steel

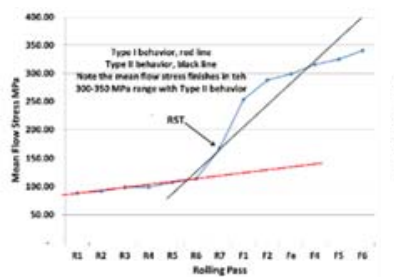

Type I $\quad$ Static
Recrystallization
and
and recrystallization $(75 \%$ total), $\mathrm{C} / \mathrm{Mn} / \mathrm{Nb}$ Steel
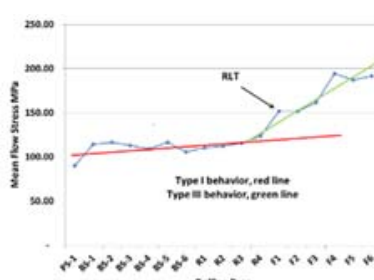

Type I Static Recrystallization $(77 \%$ total) and Type III Metadynamic Recrystallization $(75 \%$ total), $\mathrm{C} / \mathrm{Mn} / \mathrm{Nb}$ Steel

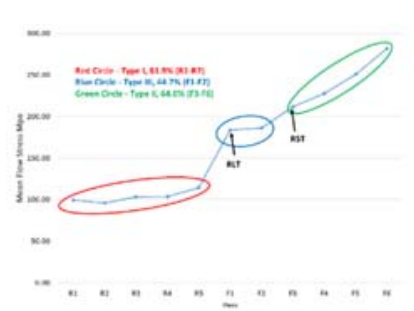

Type I Static Recrystallization $(82 \%$ total), Type III Metadynamic

Recrystallization $(45 \%$ total) and Type II NoRecrystallization (64\% total), $\mathrm{C} / \mathrm{Mn} / \mathrm{Nb} / \mathrm{Ti}$ Steel

Figure 14. Examples of actual production data mean flow stress for various alloy and processing designs showing various recrystallization behavior types. Note the general shape of the curves along with the ranges of mean flow stress strengths.

Figure 15 shows examples of two different products rolled with a $\mathrm{C} / \mathrm{Mn} / \mathrm{V} / \mathrm{Nb}$ alloy design in 50 and $65 \mathrm{~mm}$ thicknesses. Note the different volume fractions of recrystallization behaviors as seen in the actual mill mean flow stress data and the corresponding $1 / 4$ thickness and centerline thickness grain size and uniformity.

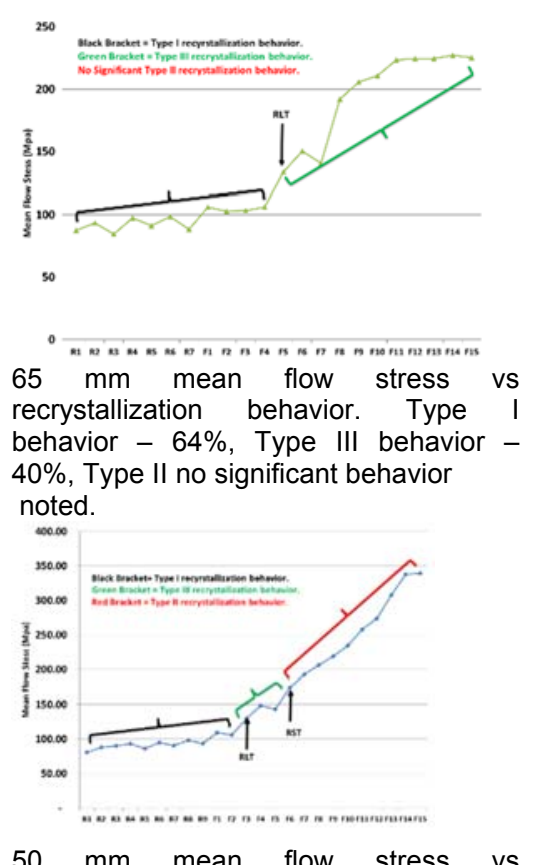

$50 \mathrm{~mm}$ mean flow stress vs
recrystallization behavior. Type I behavior $-65 \%$ total, Type III behavior $31 \%$, Type II behavior - 32\%

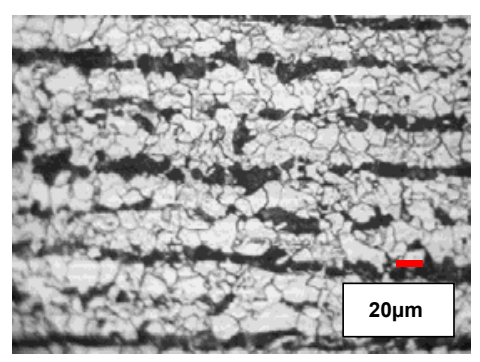

$1 / 4$ Thickness $200 x$



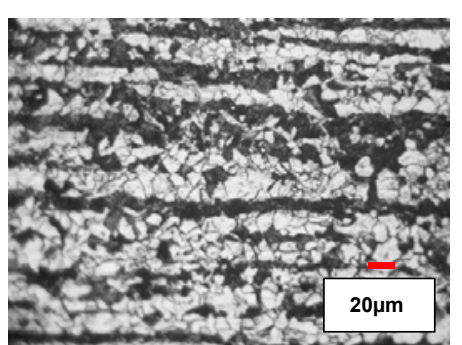

Center Thickness 200x

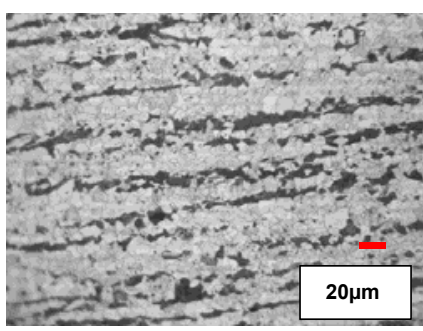

Center Thickness 200x

Figure 15. Example of mean flow stress vs. per pass recrystallization behavior and final cross sectional grain size for $65 \mathrm{~mm}$ and $50 \mathrm{~mm} \mathrm{C} / \mathrm{Mn} / \mathrm{V} / \mathrm{Nb}$ structural steel. Note the obvious difference in grain size through the cross section differences. 


\section{CONCLUSION}

Understanding the three recrystallization behaviors of structural steels is important in developing the proper/optimum metallurgy, microstructure, shape and final mechanical properties. These recrystallization behaviors and their interactions with each other determine the final cross sectional grain size and distribution. Alloy, in particular niobium, along with proper process design will determine what recrystallization behavior occurs and the final transformed ferrite cross sectional grain size. Equations from research, newly develop metallurgical models along with actual mean flow stress data generate from the rolling mill can be used to design and determine which recrystallization behavior type is being generated and the volume fraction of each type. Examples from actual production have been shown.

\section{REFERENCES}

1 Stalheim D, Glodowski R, Fundamentals of the Generation of Fine Grained As-rolled Structural Steels, Proceedings and Presentation at AIST International Symposium on the Recent Developments in Plate Steels, Winter Park, Colorado USA, June, 2011.

2 Stalheim D, A Practical Guide to Metallurgical Design for Rolled Structural Steels, Proceedings of $50^{\text {th }}$ ABM Rolling Seminar, Ouro Preto, Brazil, November, 2013.

3 Samuel F, Barbosa R, Boratto F, Yue S, Jonas J, Laboratory Simulation of Flow Stresses during Strip Rolling Using High Strain Rate Torsion Testing, Proceedings International Conference on Physical Metallurgy of Thermomechanical Processing of Steels and Other Metals (Thermec 88), Tokyo, Japan, June, 1988.

4 Barbosa R, Physical Metallurgy in the Hot Rolling of Microalloyed Steels, Proceedings of $50^{\text {th }}$ ABM Rolling Seminar, Ouro Preto, Brazil, November, 2013.

5 Cuddy L, The Effect of Microalloy Concentrations on the Recrystallization of Austenite during Hot Deformation, Thermomechanical Processing of Microalloyed Austenite, Warrendale, PA, TMS, 1982.

6 Stalheim D, Wright M, Fundamental of Developing Fine Grained Sturctures in "As Rolled" Long Products, Proceedings of $51^{\text {st }}$ ABM Rolling Seminar, Foz do Iguacu, Brazil, October, 2014.

7 Hulka K, Niobium Microalloyed Plate Products for Welded Construction, CBMM/CITIC Metal Short Course, Beijing, China, June 2006.

8 Sellars C, Hot Working and Forming Processes, The Metals Society, London, 1980.

9 Lu J, Ivey D, Henein H, Wiskel J, Omotoso O, Microstructure Characterization and Strengthening Mechanisms of Microalloyed Steels, Proceedings of $7^{\text {th }}$ ASME International Pipeline Conference, Calgary Canada, 2008.

10 Boratto F, Barbosa R, Yue S, Jonas J, Effect of Chemical Composition on the Critical Temperatures of Microalloyed Steels, Proceedings International Conference on Physical Metallurgy of Thermomechanical Processing of Steels and Other Metals (Thermec 88), Tokyo, Japan, June, 1988.

11 Bai D, Bodnar R, Ward J, Dorricott J, Sanders S, Development of Discrete X80 Line Pipe Plate at SSAB Americas, Proceedings AIST International Plate Symposium, Winter Park, CO, USA, June 2011. 